\title{
A Swarm Intelligence Networking Framework for Small Satellite Systems
}

\author{
Zijing Chen ${ }^{1}$, Yuanyuan Zeng, \\ ${ }^{1}$ The Academy of Satellite Application, Beijing, China \\ ${ }^{2}$ School of Electronic Information, Wuhan University, Wuhan, China \\ Email: zengyy@whu.edu.cn \\ Received June, 2013
}

\begin{abstract}
Recent development of technologies and methodologies on distributed spacecraft systems enable the small satellite network systems by supporting integrated navigation, communications and control tasks. The distributed sensing data can be communicated and processed autonomously among the network systems. Due to the size, density and dynamic factors of small satellite networks, the traditional network communication framework is not well suited for distributed small satellites. The paper proposes a novel swarm intelligence based networking framework by using Ant colony optimization. The proposed network framework enables self-adaptive routing, communications and network reconstructions among small satellites. The simulation results show our framework is suitable for dynamic factors in distributed small satellite systems. The proposed schemes are adaptive and scalable to network topology and achieve good performance in different network scenarios.
\end{abstract}

Keywords: Small Satellite Systems; Ant Colony Optimization; Swarm Intelligence; Network Reconstruction

\section{Introduction}

Recently, research on distributed small satellites attracts a lot of focus. Small satellite has low manufacturing and launch costs, while the network functionality is limited for distributed and dynamic topology. The low data- rate and limited transmission windows toward the ground station bring challenges for small satellite communications. The small satellite systems enable complex sensing tasks such as multipoint observation, co-observation, etc [1]. Grouping is an efficient method to cluster small satellites and manage them. The grouping of small satellites is usually executed according to the spatial characters. Some realistic examples of grouping include formation flying and constellation of small satellites for missions. In space related networking, the disruptive tolerant characteristic is a hot topic. The reconstruction capability with recovery is necessary especially for the intermittent network situation by changing orbits for small satellites in missions. The complexity of distributed small satellite systems has led the research interests of methodologies in self-organized and self-adaptive network architecture and configuration. In this paper, we present a novel swarm intelligence network framework for distributed small satellite systems by using ant colony optimization (ACO). The objective is to build a heuristic network

\footnotetext{
${ }^{*}$ Corresponding author.
}

communication framework that achieves good resource allocation with self-adaptive reconstructing ability.

Section II presents the background and related work. Section III is the system model. In Section IV, we present the ant colony optimization based small satellites network framework. Section $\mathrm{V}$ is simulations and evaluations. Section VI concludes this paper.

\section{Background and Related Work}

Recent research on the small satellite systems illustrate the advantages of distributed and inexpensive small satellites to design, build, launch and operate when compared with the traditional satellite missions. Burlacu et al [2] analyze the small satellite systems on the challenging open issues. They propose that the future hop research topic on small satellites routing; and suggest utilize the communication schemes of ad hoc networks. The distributed small satellite system mission examples include UWE-1 project. It was initiated in 2004 to develop a pico-satellite platform for telecommunication experiments [3]. The successor UWE-2 was developed in 2007 to demonstrate the small satellite capabilities in terms of attitude determination[4]. The future prospects of UWE project include the increase of robustness and rapid response by using satellite formations and swarms for advanced space missions.

Many researchers envision satellite networking topol- 
ogy technologies as a promising approach to realize new innovative collaborative space missions. The main topology technologies of distributed satellite systems include formations, constellations, clusters and swarms.

Formation is a cost-effective system approach that solves the problem of the size and mass for a single satellite with limited resources. Formation maintains the relative distance between entities in the same or very similar orbit. Braukhane et al [5] propose a scalable formation flying system named FormSat, which consists of transparent communication satellites connected via inter-satellite links to a hub satellite by allowing in-orbit resources management and optimization.

Constellation is a multi-satellite system with the entities distributed in different orbits. The Global Positioning System (GPS) and Iridium satellite constellation are two classical examples of constellation. Surrey Satellite Technology Ltd. designed, built and launched the world's first constellation to provide daily global earth observation coverage with the telecommunication and earth disaster monitoring constellation[6].

Clusters and swarms are methods for small satellite to cooperate with each other and achieve a common goal. Qin et al propose a weight based dominating set clustering algorithm for small satellite networks to group the satellites by the spatial characteristics. Swarm schemes are originated from flocks, schools, etc. The swarm based networking topology help the communications and tasks achieve global optimization from the convergence of individual behaviors.

Recently, another emerging topic in distributed space missions named fractional spacecraft is proposed. The American Defense Advanced Research Projects Agency announced F6 system program aiming at the feasibility and benefits of fractionated satellite that fulfilled with a fractionated cluster of free-flying, wireless interconnected modules[7].

Our work will utilize the swarm intelligence and build a resource optimized network framework that enable low-cost communications and reconstructing capability for in-orbit satellites considering intermittent network scenarios.

\section{System Model}

In the paper, we consider about the Low Earth Orbit (LEO) small satellite. As the small satellite fly close to earth, usually between $500 \mathrm{~km}$ and $850 \mathrm{~km}$ altitude, we approximate the orbit as a circular model other than the ellipse orbit to simply the problem formulation. In this case, the satellite moves in an even circular orbit model. An ad hoc networking can be used for small satellite network framework building while each satellite is a node. A specific ID is used to denote it. To achieve the resource optimization when building the framework, each node collects the neighborhood information by periodic HELLO messages: the neighbor satellite ID, neighbor inclination Inc, right ascension of the ascending neighbor $R a$, the orbit information $O r b$, the time of duration with the neighbor Dur, and the neighbor resource metric Res.

In the networking framework, each node will try to search the optimized node as the neighborhood and form a resource optimized network architecture for communications in missions. According to this, the stability is one of the main factors in building the framework. The relative static satellite is preferred to form neighborhood with temporal and spatial stability. The same orbital node or the node with similar orbit is considered. For the satellite within a short distance, we can still approximate consider their position as static, i.e., for two satellite $i$ and $j$, if $d_{i j}<d_{\max }$ within $\operatorname{Dur}(i, j)$, we say $i$ and $j$ keeping stability. The distance can be calculated by:

$$
d_{i j}=\sqrt{R_{i}^{2}+R_{j}^{2}-2 R_{i} R_{j} \cos \theta_{i j}}
$$

In Formula (1), $R_{i}$ and $R_{j}$ denote the orbit radius of node $i$ and $j$, respectively. $\theta_{i j}$ is the angle of the two nodes towards the earth.

Beyond the stability, the resource optimization is another factor in the framework. The neighbor resource metric Res can be defined as the weighted combinations of power, link data rate, and the maximal time window length toward the earth ground station with the unit as time slots.

Our motivation is to build a ACO-based small satellite networking topology and communication routing framework to form the network topology and communication links among satellites that achieve the global resource optimization.

\section{ACO Based Network Framework Schemes}

\subsection{Network Framework Using ACO}

In this paper, we utilize Ant Colony Optimization[8] to form and provide a self-organized network framework for communication among small satellite. The framework provides autonomous inter-satellite communications and self-adaptive topology with reconstruction capability when part of the link fails. To achieve this, the virtual agents (artificial ants) in ACO algorithm is utilized to search the optimal solutions based on decentralized coordination among the artificial ants. During the candidate solution searching process, each artificial ant $k$ on current hop $i$ will decide the outgoing link toward node $j$ according to Formula (2).

$$
p_{i j}^{k}= \begin{cases}\frac{\tau_{i j}^{\alpha} \eta_{i j}^{\beta}}{\sum_{l \in N\left(s^{p}\right)} \tau_{i l}^{\alpha} \eta_{i l}^{\beta}} & \text { if } l \in N\left(s^{p}\right) \\ 0 & \text { otherwise }\end{cases}
$$


In which, $\tau_{i j}$ is the pheromone associated with the outgoing link $(i, j) . N\left(s^{p}\right)$ is the set of feasible next hop exploration set. In the packet routing, the next hop will be a node not visited yet to prevent loops. The parameter $\alpha$ and $\beta$ control the relative importance of $\tau_{i j}$ and $\eta_{i j}$. $\eta_{i j}$ is the heuristic information on link $(i, j)$, which is given by Formula (3). In which, $\gamma$ is the weight factor parameter.

$$
\eta_{i j}=\gamma \frac{1}{d_{i j}}+(1-\gamma) \operatorname{Res}_{j}
$$

After the local search for the current hop, the ant updates local pheromone of the recent link traversed according to Formula (4). In which, $\varphi$ is the decay coefficient between 0 and 1. $\tau_{0}$ is the initial setting value of the pheromone, which is defined by upper layer application. In this way, we can prevent the searching stop from the local optimization.

$$
\tau_{i j}=(1-\phi) \tau_{i j}+\phi \tau_{0}
$$

After finishing a network-scale search process, the pheromone can be updated according to the global solutions, as shown in Formula (5). This pheromone update process helps the convergence of solutions.

$$
\tau_{i j}=(1-\phi) \tau_{i j}+\frac{\phi}{C_{b e s t}}
$$

Where, $C_{\text {best }}$ is the total best path found from the current iteration with the optimized weighted combination of stability and resource metrics.

The ACO based network framework construction scheme can be illustrated as Algorithm 1. Line 1-Line 6 is network initialization with network model, pheromone and route path cost. Line 7-Line 15 is the ACO-based networking process. It consists many steps. In each step, it includes the route searching, local pheromone update and global pheromone update. Line 10 is the ant local route searching. The searching probability is decided by Formula (2). Line 11 is the local pheromone update according to the search route. Line 13-Line 14 is the global update according to the current best route path when the complete route path is found.

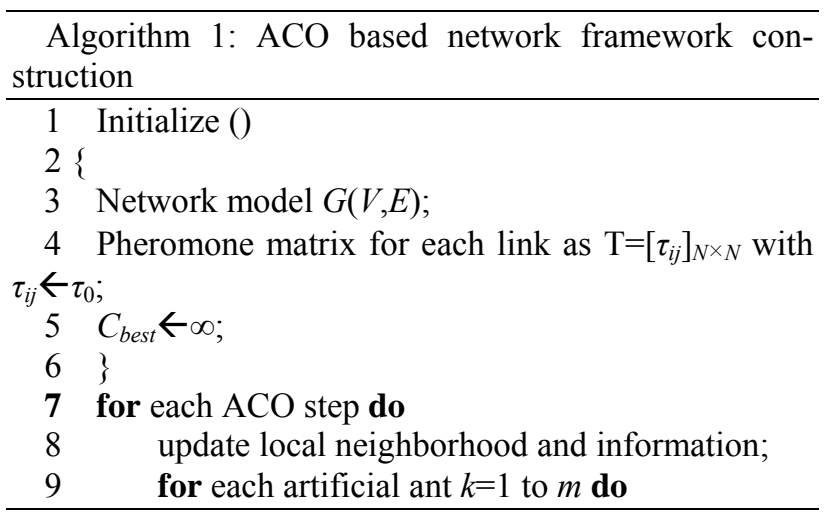

10 select the next hop $j$ according to Formula (2); 11 update $\tau_{i j}$ according to Formula (4); 12 endfor

13 update $C_{\text {best }}$ of the current step;

14 update $\tau_{i j}$ for links on the best path according to Formula (5);

15 endfor

\section{Simulations}

Our simulation is implemented by $\mathrm{C}++$. The simulation scenario is deployed in a 2-D plane, i.e., assume that all the satellites have the same inclinations and right ascensions of the ascending node. Satellites are randomly distributed from $500 \mathrm{~km}$ to $550 \mathrm{~km}$ altitude orbit. The distance between two adjacent orbits is set to $10 \mathrm{~km}$, so there are 6 orbits in total. The orbit of satellite in our simulation is illustrated as shown in Figure 1. Figure 2 shows the random position deployed in orbit of 100 small satellites. According to the satellite coverage area and earth radius, we choose $d_{\max }$ to $55 \mathrm{~km}$. We can get the generated possible links from the parameter $d_{\max }$ with distance between two small satellites that no more than $d_{\max }$. In the simulations, we consider the grounding capabilities of the satellite node (such as orbit height and distance) and use it as a metric in routing and topology formation.

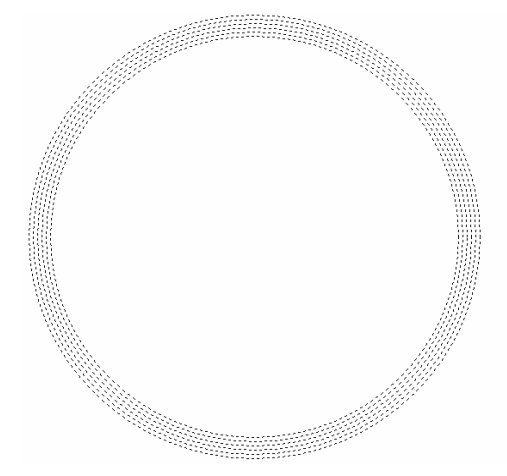

Figure 1. The orbit of LEO small satellite.

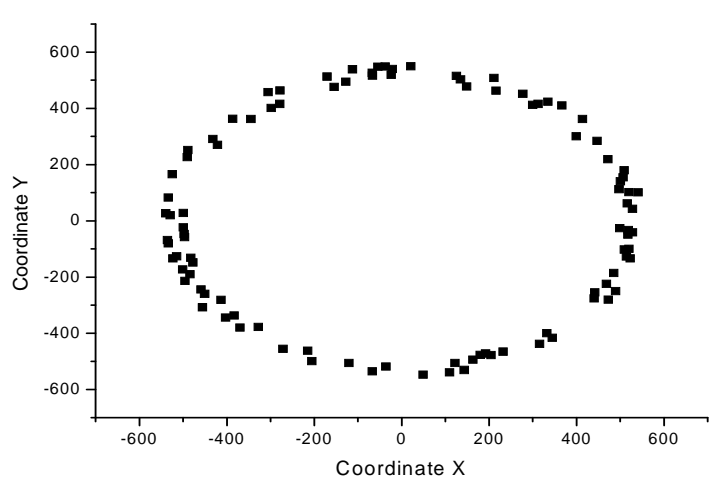

Figure 2. The random 100 satellite nodes in orbits. 
We then simulate the performance of our framework schemes in networking topology. The connection ratio is defined as the average connection degree between two nodes in the network by using our ACO based framework schemes to form the communication topology. We simulation the connection ratio as increases the number of satellite number in the network system. The results are shown in Figure 3. It shows that our framework can adaptively build the network topology with good communication connectivity especially as the node number grows big enough. The framework schemes help to maintain a network communication structure with good connectivity that benefits the distributed satellite communications among missions. Since each satellite may change the orbit around the earth to execute the missions. We then simulate the network framework with random changing orbit of node \#5 till node \#15. The results are shown in Figure 4. The results show the framework scheme can achieve a good connection ratio when the node changes the orbit. Because the scheme is intelligent to make adaptive re-routing and form the self-organized topology according to communication requirement.

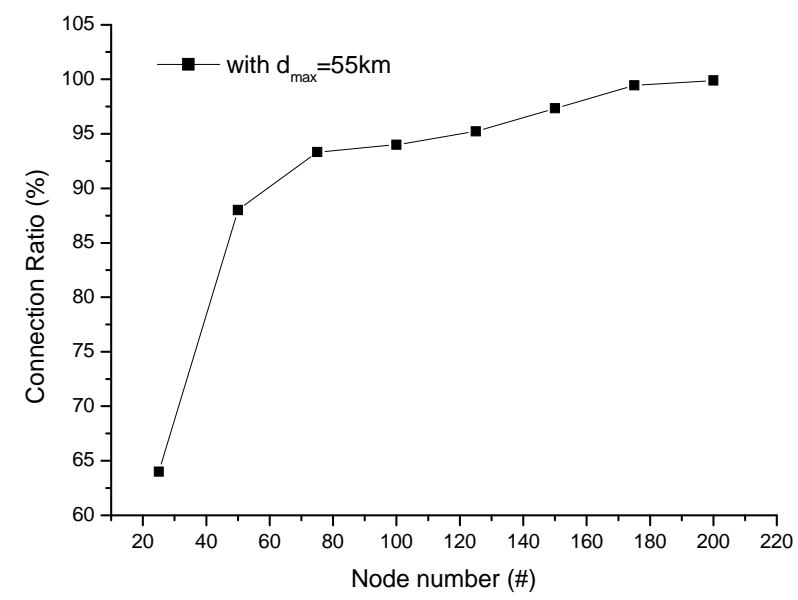

Figures 3. The connection ratio with $d_{\max }=55 \mathrm{~km}$.

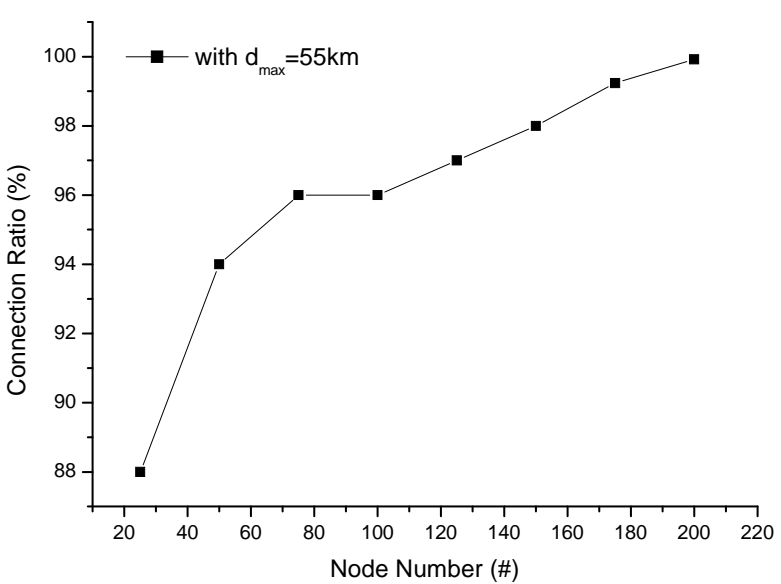

Figure 4. The connection ratio when changing the orbit.

\section{Conclusions and Future Work}

Distributed small satellite systems are the main focus for the future deep space detection and monitoring applications. The networking topology and reconstruction is challenged for the dynamic and varied space application scenarios. The traditional networking framework is not well suited for the space application-specific situations. We then propose a ACO based swarm intelligence network framework in the paper to form the heuristic, self-adaptive and self-organized network structure. The framework scheme is with fast reconstruction capability that guarantees the good performance of the small satellite systems.

We make the preliminary simulations to testify the performance of the framework schemes. We will simulate the framework scheme in different network scenarios and parameters in our future work.

\section{Acknowledgements}

This work was supported by the Open Research Fund of Scientific Research Foundation for the Returned Overseas Chinese Scholars, State Education Ministry, supported by the Open Research Fund of The Academy of Satellite Application, Chinese National Science Foundation Project (no. 61103218), The Natural Science Foundation of Jiangsu Province Youth Project (No. BK2012200), Hubei Province National Science Foundation Project (no.2011CDB446).

\section{REFERENCES}

[1] J. R. Wertz and W. J. Larson, Space Mission Analysis and Design, Space Technology Library, Microcosm Press and Springer, 3rd edition, 2007.

[2] M.-M Burlacu and P. Lorenz, "A Survey of Small Satellites Domain: Challenges, Applications, and Communication Key Issues," ICST's Global Community Magazine, 2010, pp. 1-11.

[3] M. Schmidt and F. Zeiger, "Design and Implementation of In Orbit Experiments for the Pico Satellite UWE-1," International Astronatical Congress, 2007.

[4] M. Schmidt, K. Ravandoor, O. Kurz, S. Busch and K. Schiling, "Attitude Determination for the Nano-Satellite UWE-2," Space Technology, Vol. 28, 2009, pp. 67-74.

[5] M. Arza, M. Bacher, M. Calaprice, H. Fiedler, V. Koehne, H. R. McGuire and J. J. Rivera, "FormSat, A Scalable Formation Flying Communication Satellite System," IEEE Aerospace Conference, 2010, pp. 1-18.

[6] P. Stephens, J. Cooksley, A. Curlel, L. Boland, S. Jason, J. Northham, A. Brewer, J. Anzaichi, H. Newell, C. Underwood, S. Machin, W. Sun and M. Sweeting, "Launch of The International Disaster Monitoring Constellation: The Development of a Novel International Partership in Space," Proceedings of RAST: Recent Advances in Space Technologies, 2003, pp. 525-535. 
[7] J. M. Lafleur and J. H. Saleh, "Exploring the F6 Fractionated Spacecraft Trade Space with GT-FAST," Proceedings of AIAA, 2009.

[8] M. Dorigo, M. Birattari and T. Stutzle, “Ant Colony Op- timization - Artificial Ants as a Computational Intelligence Technique," IEEE Computational Intelligence Magazine, 2006, pp. 28-39. doi:10.1109/MCI.2006.329691 